\title{
Analyse phytogéographique des forêts d'Afrique Centrale: le cas du massif de Ngovayang (Cameroun)
}

\author{
Christelle Flore Gonmadje ${ }^{1,2, *}$, Charles Doumenge ${ }^{1}$, Terry C.H. Sunderland ${ }^{3}$, \\ Michael P.B. Balinga ${ }^{4} \&$ Bonaventure Sonké 5
}

\author{
${ }^{1}$ CIRAD, Campus International de Baillarguet, TA C-105/D, FR-34398 Montpellier Cedex 5, France \\ ${ }^{2}$ IRAD - Herbier National, BP 1601, Yaoundé, Cameroun \\ ${ }^{3}$ Centre for International Forestry Research (CIFOR), PO Box 0113, BOBCD Bogor 16000, Indonesia \\ ${ }^{4}$ Centre for International Forestry Research (CIFOR), BP 9478 Ouagadougou, Burkina Faso \\ ${ }^{5}$ Laboratoire de Botanique - Systématique et d'Ecologie, Ecole Normale Supérieure, Université de Yaoundé I, BP 047, Yaoundé, Cameroun \\ *Author for correspondence: cgonmadje@yahoo.fr
}

\section{Phytogeographical analysis of Central African forests: the Ngovayang massif (Cameroon)}

Background and aims - The Ngovayang massif, in southern Cameroon, is part of a group of small hills along the Atlantic coast of the Gulf of Guinea. This area is known for its floristic richness and its high level of endemism. We conducted a phytogeographical analysis of this massif and evaluated its floristic affinities with other forested sites in Central Africa.

Methods - Phytogeographical analysis was based on a comparison of lists of tree species with $\mathrm{dbh} \geq 10$ $\mathrm{cm}$, inventoried in 1-ha permanent plots, and established in old growth lowland forests of Central Africa. The floristic list for Ngovayang used for this analysis contained 201 species.

Key results - The lowland forests of the Ngovayang massif are dominated by LeguminosaeCaesalpinioideae, followed by Olacaceae and Burseraceae, taxa characteristic of dense old-growth Atlantic moist forests. The Ngovayang flora includes a high proportion of Guineo-Congolean species $(79 \%)$, particularly Lower Guinean species (30\%). Phytogeographical analysis showed that forested sites of central Africa are clearly separated into continental sites and the more coastal sites of the Lower Guinean domain, including Ngovayang. Within the Lower Guinean domain, the main floristic discontinuity appears to be situated in southern Cameroon-northern Equatorial Guinea. Ngovayang and Takamanda, which are situated on opposite sides of the Cameroon volcanic line, show stronger floristic affinities with each other than either does with other Lower Guinean forest sites located to the south of the Ngovayang massif.

Conclusions - Ngovayang may constitute a transition zone between Nigerian forests and those ranging from southern Cameroon to the Democratic Republic of the Congo. For tree species of the Lower Guinean domain, the climatic hinge separating boreal and austral climates appears to be a more important phytogeographic barrier than the Cameroon volcanic line or the Sanaga River.

Key words - Central Africa, Ngovayang, phytogeography, climatic hinge, 1-ha permanent plots.

Objectifs - Le massif de Ngovayang (Sud-Cameroun) fait partie des petits sommets qui longent le golfe de Guinée. Cette région est connue pour sa forte richesse floristique et son taux d'endémisme élevé. Ce travail a pour objectif une analyse phytogéographique de ce massif et l'évaluation de ses affinités floristiques avec d'autres sites forestiers d'Afrique centrale atlantique.

Méthodes - L'analyse phytogéographique fut basée sur la comparaison des listes d'espèces d'arbres de $\mathrm{dhp} \geq 10 \mathrm{~cm}$, issues de parcelles permanentes de 1-ha établies dans des forêts matures de basse et moyenne altitude d'Afrique centrale atlantique. La liste floristique du massif de Ngovayang retenue pour cette analyse comprend 201 espèces d'arbres.

Résultats-clés - Les forêts de basse altitude du massif de Ngovayang sont dominées par les LeguminosaeCaesalpinioideae, suivies des Olacaceae et des Burseraceae, tous taxons caractéristiques des forêts matures denses humides atlantiques. La flore de Ngovayang compte une forte proportion d'espèces 
guinéo-congolaises $(79 \%)$ et plus particulièrement d'espèces guinéennes inférieures $(30 \%)$. L'analyse phytogéographique montre qu'il existe dans la région étudiée une séparation nette entre les sites continentaux et les sites côtiers du domaine bas-guinéen, y compris le massif de Ngovayang. A l'intérieur du domaine bas-guinéen, la discontinuité floristique la plus importante apparaît aux confins du Sud-Cameroun et du Nord de la Guinée-Equatoriale. Ngovayang et Takamanda, situés de part et d'autre de la ligne volcanique du Cameroun, présentent de plus fortes affinités floristiques entre eux qu'avec les autres forêts situées au sud du massif de Ngovayang.

Conclusion - Ngovayang pourrait constituer une zone de transition importante entre les forêts du Nigéria et celles s'étendant du Sud-Cameroun jusqu'en République Démocratique du Congo. La charnière climatique séparant les climats boréal et austral semble constituer une barrière phytogéographique plus importante pour les arbres des forêts de basse altitude que la ligne volcanique du Cameroun ou le fleuve Sanaga.

\section{INTRODUCTION}

Les forêts tropicales humides africaines font partie des écosystèmes les plus importants, les plus complexes et les plus riches de la planète (Wilson 1995, van Gemerden 2004). La région guinéo-congolaise, qui couvre une superficie de 2,8 millions de $\mathrm{km}^{2}$, s'étend du sud du Sénégal à l'ouest de l'Ouganda et au nord de l'Angola, et renferme le principal massif de forêts denses humides d'Afrique, avec plus de $80 \%$ de plantes endémiques (White 1983). Ces forêts présentent une grande gamme de variation, tant en termes de phénologie (forêts sempervirentes à semi-décidues) que de composition floristique. Au sein de cet ensemble, les deux plus importantes subdivisions floristiques correspondent, d'une part, aux forêts situées à l'intérieur et en périphérie de la cuvette congolaise - les forêts 'congolaises' - et, d'autre part, à celles situées en Afrique occidentale et en Afrique centrale atlantique, les forêts 'guinéennes'.

Ces forêts guinéennes sont séparées en deux blocs par l'avancée des savanes jusqu'à la côte atlantique, au Togo et au Bénin: un domaine floristique 'haut-guinéen' ou guinéen supérieur (Afrique occidentale) et un domaine 'bas-guinéen' ou guinéen inférieur (Afrique centrale autour du Golfe de Guinée; White 1979). Les forêts sempervirentes côtières, le long du littoral atlantique africain, de la Sierra Leone jusqu'à l'ouest du Gabon, bénéficient des climats les plus humides, avec des précipitations annuelles qui peuvent dépasser $2000 \mathrm{~mm}$. Ces forêts renferment une flore très riche avec un taux d'endémisme élevé (Aubréville 1958, 1962, Hall \& Swaine 1976, 1981, White 1983, Letouzey 1985, Hawthorne 1995, Doumenge et al. 2003).

Le domaine bas-guinéen a été à son tour subdivisé en deux secteurs phytogéographiques: le secteur du bassin sédimentaire côtier (forêts littorales) et celui des collines atlantiques (forêts biafréennes de Letouzey 1968, 1985, Caballé 1978, Doumenge et al. 2001). Ces collines atlantiques forment un archipel qui borde, en retrait, le littoral atlantique depuis le Cameroun jusqu'en République Démocratique du Congo (RDC). Les principaux sommets n'y dépassent guère 1000 m d'altitude, et incluent: les monts Rumpi, le massif de Ngovayang et la région de Campo-Ma'an (Cameroun), la région du Monte Alèn et du Monte Mitra (Guinée Equatoriale), les monts de Cristal et les monts Doudou (Gabon), le massif du Chaillu (Gabon et Congo) ainsi que la chaîne du Mayombe (du Gabon à la RDC; fig. 1).
Si les schémas biogéographiques développés par certains auteurs (Monod 1957, Lebrun 1960, 1961, White 1983) restent tout à fait d'actualité à l'échelle du continent, ils se montrent largement perfectibles à l'échelle de secteurs phytogéographiques plus restreints comme le bas-guinéen. Des études phytogéographiques plus fines ont été réalisées à l'échelle des pays, à l'instar de Letouzey $(1968,1985)$ pour le Cameroun, Caballé (1978) pour le Gabon, Senterre (2001) pour la Guinée équatoriale et Lubini $(1997,2001)$ pour la RDC. Certains de ces travaux sont basés sur la chorologie (répartition des espèces), d'autres sur des inventaires écologiques ou phytosociologiques. Les travaux de synthèse à l'échelle de la région guinéo-congolaise ou de ses subdivisions, basés sur des méthodes d'échantillonnage, quantifiées et comparables, font encore largement défaut. La seule tentative, récente, est celle de Senterre (2005), qui a comparé plusieurs forêts le long d'un méga-transect partant de l'océan atlantique jusque dans la Réserve de biosphère du Dja. Ce travail donne un premier aperçu quantifié de la variation du peuplement arboré de ces forêts, mais reste encore très insuffisant pour une comparaison exhaustive des affinités floristiques sur l'ensemble du secteur floristique guinéen, voire bas-guinéen.

Par ailleurs, des études biogéographiques ou des révisions taxonomiques limitées à certains groupes systématiques, encore trop peu nombreuses, apportent aussi un éclairage complémentaire dans l'analyse des affinités floristiques entre les forêts de cette région. Il s'agit des travaux sur certaines familles telles que les Euphorbiaceae (Léonard 1965) et les Orchidaceae du domaine bas-guinéen (Droissart 2009), la révision des genres Annickia (Annonaceae; Versteegh \& Sosef 2007), Anthonotha (Leguminosae-Caesalpinioideae; Breteler 2010), Baphia (Leguminosae-Faboideae; Soladoye 1985), Begonia (Begoniaceae; Sosef 1994) ou Diospyros (Ebenaceae; White 1978). Il ressort néanmoins de ces travaux que tous les petits reliefs bordant la côte atlantique possèdent en commun une caractéristique: l'abondance de LeguminosaeCaesalpinioideae grégaires (White 1983, Letouzey 1985, Senterre 2001, 2005). S'il est établi qu'il existe des différences entre les forêts continentales du domaine congolais et celles, plus côtières, du domaine guinéen (Letouzey 1968, White 1983, Senterre 2005), la variation floristique et les affinités phytogéographiques fines, depuis les forêts bordant la ligne volcanique du Cameroun (LVC) jusqu'au Mayombe congolais demeurent mal connues. La LVC, constituée de grands sommets montagneux (Mont Cameroun, Mont Oku, 


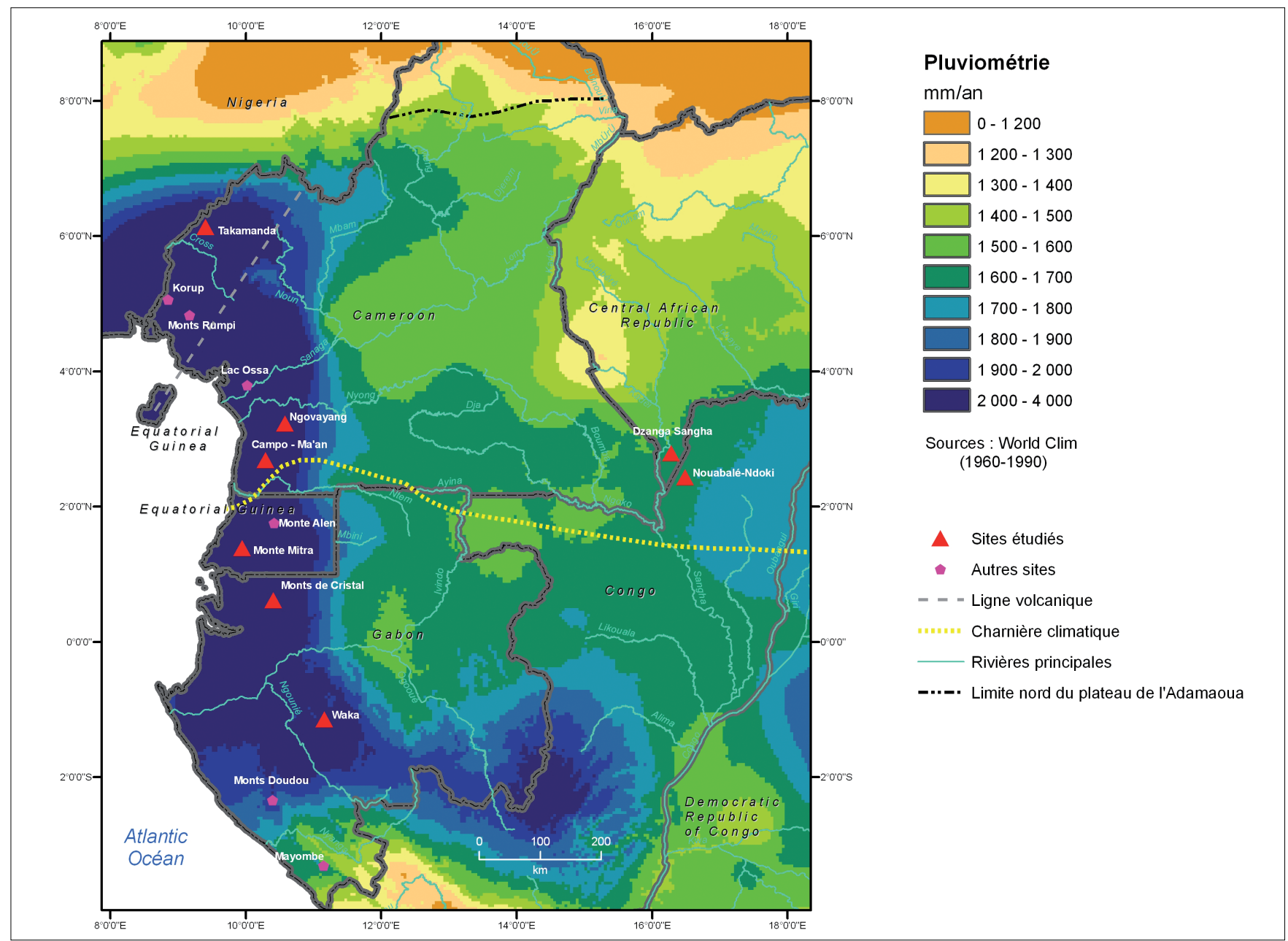

Figure 1 - Localisation du massif de Ngovayang et des autres sites étudiés en Afrique Centrale. Charnière climatique adaptée d'après Suchel (1990). Carte réalisée par le Cirad en Janvier 2012.

Mont Kupé...), et le fleuve Sanaga ont-ils pu jouer le rôle de barrière entre les forêts des confins nigéro-camerounais et celles situées à l'Est de la LVC et plus au Sud? Le basculement climatique qui se produit à l'extrême Sud du Cameroun, entre climat d'hémisphère Nord et climat d'hémisphère Sud, matérialisé par la 'charnière climatique' (Saint-Vil 1984, Suchel 1990, Fontaine \& Bigot 1993, Wotling et al. 1995, Bigot et al. 1997, Thomas \& Goudie 2000, Balas et al. 2007), influence-t-il la composition floristique de ces forêts?

Le massif forestier de Ngovayang (alt. 50-1090 m), situé à la charnière entre la LVC et l'ensemble des collines descendant du Sud-Cameroun jusqu'en RDC, est idéalement situé pour tenter de répondre à ces questions et nous permettra d'explorer les relations phytogéographiques entre les forêts de tout cet ensemble floristique bas-guinéen. Par ailleurs, ce massif, bien qu'il ait fait l'objet d'une étude phytogéographique préliminaire (Letouzey 1985), demeure toujours peu exploré. Les premiers travaux d'inventaires botaniques, très localisés, concernaient uniquement des collectes botaniques (en particulier celles de Zenker, entre 1896 et 1922). D'autres études, plus récentes, ont porté sur les collections de spécimens botaniques nécessaires à l'étude de certains groupes taxonomiques, notamment les Orchidaceae (Droissart 2009) et les Rubiaceae (Sonké et al. 2006a, 2006b, 2008). Aucun inventaire quantifié de la végétation n'avait été réalisé avant la présente étude.

Sur la base d'inventaires quantifiés des peuplements arborés, nous nous proposons donc d'analyser la position phytogéographique du massif de Ngovayang et de préciser ses affinités floristiques avec d'autres massifs forestiers d'Afrique Centrale, en particulier dans le secteur floristique de Basse-Guinée.

\section{MÉTHODES}

\section{Site d'étude}

Le massif montagneux de Ngovayang se situe dans le Sud du Cameroun, à environ $80 \mathrm{~km}$ de la côte atlantique. Il s'étend sur 102000 ha, entre les parallèles $3^{\circ} 12^{\prime}-3^{\circ} 25^{\prime} \mathrm{N}$ et les méridiens $10^{\circ} 30^{\prime}-10^{\circ} 45^{\prime} \mathrm{E}$ (fig. 1). L'altitude varie entre $50 \mathrm{~m}$, dans la partie sud-ouest, et $1090 \mathrm{~m}$ dans la partie centre-est, sur les plus hauts sommets. La géomorphologie du massif est très complexe, constituée d'un ensemble de crêtes disséquées d'orientation principale sud-ouest/nord-est. Le versant sud-est est caractérisé par des pentes abruptes tandis que des piedmonts d'altitudes variées occupent le versant 
nord-ouest. Le socle précambrien constitue le soubassement géologique du massif, caractérisé par des roches métamorphiques acides (gneiss, migmatites, quartzites et schistes) traversées par des intrusions de roches basiques et de vieilles roches volcaniques tels que les diorites et les gabbros (Franqueville 1973).

Le climat est de type sub-équatorial (Suchel 1972). Les précipitations moyennes annuelles sont de l'ordre de $2000 \mathrm{~mm}$ (Waterloo et al. 2000). Elles diminuent avec l'augmentation de la distance par rapport à l'océan de $2836 \mathrm{~mm} \pm 393$ à Kribi, situé environ $80 \mathrm{~km}$ à l'ouest de l'extrémité sud-ouest du massif de Ngovayang, à $2150 \mathrm{~mm}$ \pm 286 à Eséka (environ $15 \mathrm{~km}$ au nord de l'extrémité nordest du massif; Olivry 1986). Deux minima et maxima pluviométriques annuels distincts sont observés et sont associés avec les mouvements nord-sud de la zone de convergence intertropicale (ZCIT): une petite saison sèche (mi-mai à miaoût) et une grande saison sèche (mi-novembre à mi-mars) qui séparent les deux saisons des pluies (mi-mars à mi-mai pour la petite saison des pluies et mi-août à mi-novembre pour la grande saison des pluies). La température moyenne annuelle est de $25^{\circ} \mathrm{C}$ (Waterloo et al. 2000). Le réseau hydrographique, très dense, est constitué de nombreuses petites rivières entrecoupées de chutes.

Les forêts atlantiques biafréennes riches en LeguminosaeCaesalpinioideae constituent le principal type de formation végétale rencontré dans la zone d'étude (Letouzey 1968, 1985). En sus des menaces globales tels que les changements climatiques, la destruction de la forêt pour des besoins en terres cultivables et l'exploitation du bois ont contribué à la destruction de la végétation naturelle depuis des décennies, principalement dans les zones basses et plates facilement accessibles (Letouzey 1985). Des forêts secondaires se sont fréquemment développées dans ces régions de basse altitude, en dessous de $300 \mathrm{~m}$. D'autres pressions humaines, telles que la chasse ou la cueillette de produits forestiers, sont aussi notables, y compris en altitude. Cependant, en raison de sa topographie accidentée et de sa faible densité démographique, le massif de Ngovayang a été jusqu'ici relativement préservé de ces menaces et de vastes étendues de forêts très peu ou non perturbées y subsistent encore.

\section{Collecte des données}

L'analyse floristique et phytogéographique a été basée sur l'inventaire du peuplement arboré de parcelles permanentes de 1 ha $(100 \mathrm{~m} \times 100 \mathrm{~m})$. Ces parcelles ont été installées dans plusieurs sites d'Afrique Centrale, à savoir: la réserve forestière de Takamanda (6 parcelles) et le massif de Ngovayang ( 5 parcelles) au Cameroun, le Monte Mitra (3 parcelles) en Guinée Equatoriale, les monts de Cristal (5 parcelles) et le parc national de Waka (2 parcelles) au Gabon, le parc national de Nouabalé-Ndoki (3 parcelles) au Congo et le parc national de Dzanga-Sangha (une parcelle) en République Centrafricaine (fig. 1).

Dans ces parcelles, tous les arbres dont le diamètre du tronc à hauteur de poitrine (dhp, mesuré à $1,3 \mathrm{~m}$ du sol pour les arbres sans contreforts et sans anomalies) était supérieur ou égal à $10 \mathrm{~cm}$ ont été identifiés et mesurés. Le protocole et les détails de mesure suivent les recommandations en vigueur (Dallmeier et al. 1992, White \& Edwards 2001). De plus en plus utilisé en Afrique Centrale, ce protocole facilite les comparaisons entre les sites. Dans le massif de Ngovayang, les spécimens d'herbier ont été récoltés pour la plupart des espèces recensées en vue de confirmer les identifications de terrain. Les identifications botaniques ont été réalisées à l'aide des flores existantes et les échantillons ont été comparés aux spécimens de référence de l'Herbier National du Cameroun. Afin d'homogénéiser les données, les listes d'espèces utilisées pour les analyses comparatives des sites étudiés sont issues uniquement des parcelles installées dans les forêts les plus matures possibles, sur terre ferme et d'altitude inférieure à $700 \mathrm{~m}$. Les forêts ayant subies un impact humain (forêts secondaires, forêts exploitées) et celles dépendantes de conditions environnementales particulières (forêts inondables et forêts d'altitude) ont été exclues de l'étude pour faciliter les comparaisons entre les différents sites.

\section{Analyse floristique}

Deux indices d'importance écologique des espèces et des familles ont été calculés pour les parcelles du massif de Ngovayang, pour lesquelles nous disposons des données d'abondance: 1'Importance Value Index (IVI; Curtis \& McIntosh 1951) et le Family Importance Value Index (FIV; Mori et al. 1983). Des détails sont donnés en matériel supplémentaire (appendice électronique 3).

\section{Analyse phytogéographique}

Les données relatives aux aires de répartition des espèces sont issues de la bibliographie, à savoir, les flores (Keay \& Hepper 1954-1972, Aubréville \& Leroy 1961-1992, 1963-1978, Hallé 1961-1987, Letouzey 1968, 1985, Lebrun \& Stork 1991-1997) et d'autres publications (White 1983, Sonké 2005, Senterre 2005, Tchouto 2004), ainsi que les bases de données en ligne (Conservatoire du Jardin Botanique de Genève: http://www.ville-ge.ch/musinfo/bd/cjb/africa/recherche.php).

L'analyse phytogéographique a porté sur 201 taxons déterminés au niveau de l'espèce. Les différents types d'aires de répartition retenus sont basés sur la classification de White (1979, 1983).

\section{A. Espèces à large distribution}

Cosmopolites: espèces réparties dans le monde entier, aussi bien dans les zones tempérées que tropicales;

Pantropicales: espèces présentes en Afrique, Amérique et Asie tropicales;

Afro-américaines: espèces répandues en Afrique et Amérique tropicales;

Paléotropicales: espèces connues en Afrique et Asie tropicales ainsi qu'à Madagascar et en Australie;

Afromalgaches: espèces communes aux îles de la région malgache et de l'Afrique continentale.

B. Espèces de liaison

Espèces dont l'aire de distribution couvre deux ou plusieurs régions floristiques africaines et qui sont trop largement répandues ou trop abondantes par endroits pour être considérées comme des sub-endémiques. Ces éléments 
sont soit des transgresseurs chorologiques et écologiques, soit simplement des espèces à large distribution et à écologie assez uniforme.

C. Espèces sub-endémiques guinéo-congolaises

Espèces qui s'étendent aux zones de transitions adjacentes ou qui débordent légèrement au-delà de ces dernières en tant qu'espèces d'intrusion marginale ou en tant qu'espèces formant des peuplements satellites faiblement éloignés.

D. Espèces endémiques guinéo-congolaises

Omni-guinéo-congolaises: présentes dans toute la région guinéo-congolaise;

Omni-guinéennes: présentes dans les domaines guinéens inférieur et supérieur;

Centro-guinéo-congolaises: espèces dont la distribution couvre les domaines guinéen inférieur (du sud du Nigeria à la République Démocratique du Congo) et congolais;

Guinéennes inférieures: espèces répandues dans le souscentre guinéen inférieur, du sud du Nigeria au sud de la République du Congo;

Endémiques camerounaises: espèces exclusivement camerounaises.

La nomenclature botanique utilisée est celle adoptée par Lebrun \& Stork (1991-1997), afin de pouvoir comparer les résultats avec la bibliographie, en particulier lorsqu'il s'agit de certaines familles importantes (Olacaceae et Euphorbiaceae) qui ont été éclatées dans le système de classification d'APGIII, ou d'autres qui ont été mises ensemble (Bombacaceae, Sterculiaceae et autres, dans la famille Malvaceae sensus APGIII). Les autorités taxonomiques de toutes les espèces nommées dans cette étude sont données en matériel supplémentaire (appendices électroniques $1 \& 2$ ).

La classification des relevés a été réalisée en fonction de l'indice de dissimilarité de Bray-Curtis binaire $\left(\mathrm{BC}_{\mathrm{ij}}\right)$ en utilisant la bibliothèque Vegan du logiciel R 2.10.1 (Oksanen et al. 2010); des détails sont donnés en matériel supplémentaire (appendice électronique 3).

Cette première analyse nous a permis d'identifier des groupes de relevés proches sur la base d'occurrence d'espèces (données de présence / absence), à partir des listes d'espèces inventoriées dans chaque site. La distance entre les relevés varie de 0 (relevés identiques, compositions floristiques identiques) à 1 (relevés sans recouvrement, compositions floristiques complètement différentes, aucune espèce commune).

Une classification ascendante hiérarchique sur la base des méthodes agglomératives à liens complets, a été utilisée pour la construction du dendrogramme de ressemblances entre les relevés.

\section{RESULTATS}

\section{Richesse et composition floristique globale des forêts de Ngovayang}

Les cinq parcelles installées dans les forêts de basse altitude du massif forestier de Ngovayang ont permis de recenser au total 2658 individus à $\mathrm{dhp} \geq 10 \mathrm{~cm}$ correspondant à 293 espèces et morpho-espèces, 170 genres et soixante familles.
Tableau 1 - Spectre phytogéographique des espèces du massif de Ngovayang.

Les grands groupes phytogéographiques sont précédés des lettres A, B , C et D. Ils sont classés du type le plus large au plus restreint.

\begin{tabular}{lcc}
\hline Type phytogéographique & $\begin{array}{c}\text { Nombre } \\
\text { d'espèces }\end{array}$ & $\begin{array}{c}\text { Proportion } \\
(\%)\end{array}$ \\
\hline A. Espèces à large distribution & $\mathbf{4}$ & $\mathbf{2}$ \\
Cosmopolites & 0 & 0 \\
Pantropicales & 0 & 0 \\
Paléotropicales & 0 & 0 \\
Afro-américaines & 2 & 1 \\
$\quad$ Afro malgaches & 2 & 1 \\
B. Espèces de liaison & $\mathbf{2 0}$ & $\mathbf{1 0}$ \\
C. Espèces sub-endémiques & $\mathbf{1 8}$ & $\mathbf{9}$ \\
guinéo-congolaises & $\mathbf{1 5 9}$ & $\mathbf{7 9}$ \\
D. Espèces guinéo-congolaises & 34 & 17 \\
$\quad$ Omni-guinéo-congolaises & 45 & 22 \\
Centro-guinéo-congolaises & 17 & 8 \\
Omni-guinéennes & 60 & 30 \\
Guinéennes inférieures & 3 & 2 \\
$\quad$ Endémiques camerounaises & $\mathbf{2 0 1}$ & $\mathbf{1 0 0}$ \\
Nombre total d'espèces & & \\
\hline
\end{tabular}

Parmi ces 293 espèces, 201 ont été déterminées au niveau spécifique, 82 au niveau du genre et dix espèces restent indéterminées.

Les familles les plus riches en espèces sur la base des 293 taxons sont les Euphorbiaceae (36 espèces, soit $12 \%$ de la flore), la sous-famille des Leguminosae-Caesalpinioideae (23 espèces, soit $8 \%$ de la flore) et les familles des Ebenaceae (19 espèces), des Annonaceae (quatorze espèces), des Rubiaceae (treize espèces), des Olacaceae (onze espèces) et des Clusiaceae (dix espèces). Les Leguminosae-Caesalpinioideae représentent la (sous-)famille la plus importante en termes de FIV. Elle est suivie de près et en valeur décroissante par la famille des Euphorbiaceae, des Clusiaceae, des Myristicaceae, des Olacaceae, des Ebenaceae, des Annonaceae et des Burseraceae. On note aussi la présence des Leguminosae-Caesalpinioideae grégaires, représentées surtout par Didelotia letouzeyi, Guibourtia tessmannii et Tetraberlinia bifoliolata (Gonmadje et al. 2011).

\section{Spectres phytogéographiques des forêts de Ngovayang}

L'analyse phytogéographique des forêts de basse et moyenne altitude du massif de Ngovayang montre que les espèces guinéo-congolaises sont largement majoritaires dans le fond floristique de la zone d'étude avec $79 \%$ des espèces (tableau 1). A part un très petit pourcentage d'espèces à large répartition $(2 \%)$, les $19 \%$ restants sont soit des espèces subendémiques au centre d'endémisme guinéo-congolais, soit des espèces de liaison avec les régions phytogéographiques adjacentes. Au sein de ces espèces guinéo-congolaises, les deux principaux groupes renferment, soit des espèces endémiques du domaine guinéen inférieur, soit des espèces 
centro-guinéo-congolaises. Ces deux groupes constituent à eux seuls environ $52 \%$ de la flore.

Les espèces du domaine guinéen inférieur, caractéristiques des forêts entourant le golfe de Guinée, représentent $30 \%$ de la flore de Ngovayang. Parmi les espèces les plus importantes en termes d'IVI, on peut citer Tetraberlinia bifoliolata (Leguminosae-Caesalpinioideae), Treculia obovoidea (Moraceae), Garcinia lucida (Clusiaceae), Didelotia letouzeyi (Leguminosae-Caesalpinioideae), Oubanguia alata (Scytopetalaceae), Cola rostrata (Sterculiaceae), Sibangea similis (Euphorbiaceae), Heisteria zimmereri (Olacaceae) et Garcinia mannii (Clusiaceae). Dans ce groupe, une espèce endémique camerounaise a été collectée: Tetraberlinia korupensis (Leguminosae-Caesalpinioideae). Avant la présente étude, cette dernière espèce n'était connue que du parc national de Korup. Les familles les plus représentées sont les Euphorbiaceae ( $10 \%$ des espèces de Basse-Guinée présentes sur le site), les Sterculiaceae (7\%), les Annonaceae (6\%) les Rubiaceae (5\%) et les Leguminosae, essentiellement la sousfamille des Caesalpinioideae (9\%).

Les espèces centro-guinéo-congolaises représentent $22 \%$ de la flore et sont surtout représentées par Drypetes gossweileri, Mareyopsis longifolia, Uapaca staudtii, Dichostemma glaucescens (Euphorbiaceae), Diospyros crassiflora, D. iturensis (Ebenaceae), Aidia micrantha, Aulacocalyx jasminiflora (Rubiaceae), Pentadesma grandifolia, Allanblackia floribunda (Clusiaceae), Aphanocalyx cynometroides, Gilbertiodendron ogoouense (Leguminosae-Caesalpinioideae), et Coelocaryon preussi (Myristicaceae). Les Euphorbiaceae ( $27 \%$ des espèces), les Ebenaceae $(9 \%)$, les Rubiaceae $(9 \%)$ les Clusiaceae $(6 \%)$ et les Leguminosae-Caesalpinioideae $(6 \%)$ sont les mieux représentées.

Les espèces omni-guinéo-congolaises représentent $17 \%$ de la flore avec des espèces telles que Mareya micrantha (Euphorbiaceae), Strombosia grandifolia (Olacaceae), Anthonotha fragrans (Leguminosae-Caesalpinioideae), Oncoba glauca (Flacourtiaceae) et Cola lateritia (Sterculiaceae). Les familles des Olacaceae (12\% des espèces), des Euphorbiaceae $(9 \%)$, des Flacourtiaceae (9\%), des Sterculiaceae (9\%) et la sous-famille des Leguminosae-Caesalpinioideae (9\%) sont les plus représentées dans ce type phytogéographique.

Environ 9\% des espèces sont subendémiques guinéocongolaises. Parmi elles, on peut citer: Diospyros gabunensis (Ebenaceae), Hymenostegia afzelii (Leguminosae-Caesalpinioideae), Trichilia prieureana (Meliaceae), Discoglypremna caloneura (Euphorbiaceae) et Xylopia aethiopica (Annonaceae).

\section{Affinités floristiques avec divers autres sites d'Afrique centrale}

La classification des espèces des différents sites étudiés permet de distinguer deux groupes principaux au seuil de dissimilarité de $96 \%$ (fig. 2):

(1) un groupe formé par les deux sites continentaux: Dzanga-Sangha et Nouabalé-Ndoki,

(2) un groupe formé par les cinq sites côtiers, du domaine guinéen inférieur. Dans ce groupe, on peut distinguer, à partir de $76 \%$ de dissimilarité, deux sous-groupes. Le premier est constitué des sites situés au sud du massif de Ngovayang (monts de Cristal, Waka et Monte Mitra) et le second est formé par Ngovayang et Takamanda.

Parmi les taxa qui permettent de différencier les deux groupes sus-cités, les Leguminosae-Caesalpinioideae sont les plus représentés: Berlinia craibiana, Didelotia letouzeyi, Guibourtia tessmannii, Leonardoxa africana et Tetraberlinia bifoliolata sont uniquement présents dans les sites du domaine guinéen inférieur. D'autres espèces sont également typiques des forêts de Basse-Guinée, telles que Poga oleosa (Anisophylleaceae), Calpocalyx dinklagei (LeguminosaeMimosoideae) et Dacryodes buettneri (Burseraceae).

Parmi les espèces du domaine guinéen inférieur recensées dans les divers sites, Allophylus bullatus (Sapindaceae), Cleistanthus letouzeyi, Drypetes molunduana et Sibangea similis, (Euphorbiaceae), Cola anomala, C. megalophylla et $C$. semecarpophylla (Sterculiaceae), et Tetraberlinia korupensis (Leguminosae-Caesalpinioideae) sont communes au massif de Ngovayang et à Takamanda mais elles n'atteignent pas les sites plus au Sud (monts de Cristal, Monte Mitra et Waka). Deux sous-espèces de Leonardoxa africana (Leguminosae-Caesalpinioideae) sont également présentes dans les sites du Nord: Leonardoxa africana subsp. gracilicaulis à Ngovayang et Leonardoxa africana subsp. letouzeyi à Takamanda. Seule, cette dernière est restreinte aux confins camerouno-nigérians, la sous-espèce gracilicaulis s'étendant au Sud vers la Guinée-Equatoriale et le Gabon (McKey 2000, Brouat et al. 2004). Par ailleurs, d'autres espèces ne sont présentes que dans les sites les plus au Sud; elles n'atteignent pas Ngovayang ni Takamanda. Il s'agit, par exemple, de $B i$ kinia durandii, Daniellia soyauxii, Hymenostegia pellegrinii, et Julbernardia brieyi (Leguminosae-Caesalpinioideae), Engomegoma gordonii et Strombosiopsis sereinii (Olacaceae), et Aucoumea klaineana (Burseraceae).

Lorsque l'on réalise le même type de comparaison au niveau des familles, la classification des relevés permet également de distinguer, à partir de $44 \%$ de dissimilarité, les deux mêmes groupes principaux observés précédemment

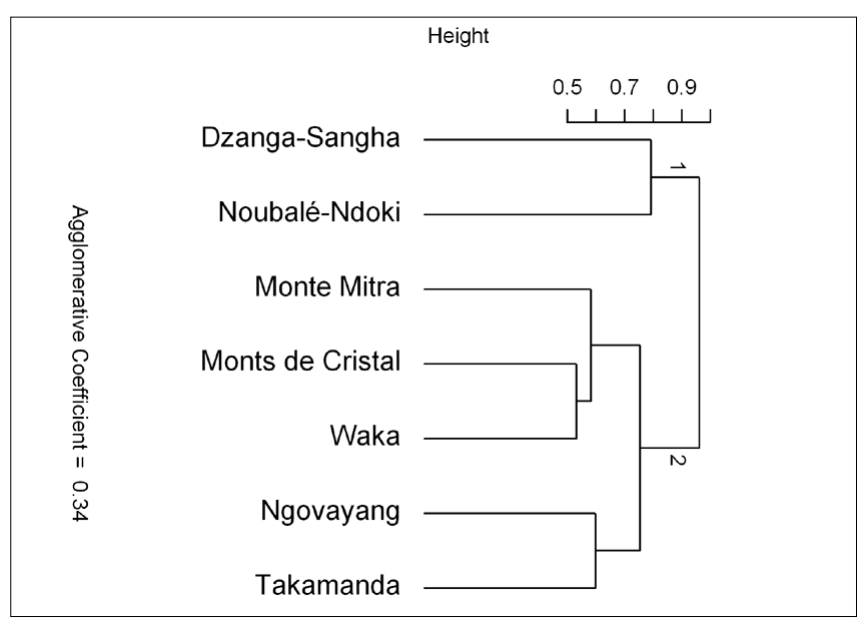

Figure 2 - Distances écologiques (Bray-Curtis binaire) entre les sites en Afrique Centrale sur la base des listes d'espèces. Le chiffre 1 représente les sites continentaux, et le chiffre 2 correspond aux sites côtiers. 
(forêts continentales vs forêts côtières), avec la même subdivision parmi les forêts périphériques au golfe de Guinée (fig. $3)$. Les indices de dissimilarité de Bray-Curtis sont globalement plus faibles, avec des valeurs inférieures à $50 \%$. Ces faibles distances traduisent une plus grande similarité floristique au niveau taxonomique des familles.

\section{DISCUSSION}

\section{Analyse phytogéographique du massif de Ngovayang}

Les proportions d'espèces guinéo-congolaises $(79 \%)$ et de liaison $(10 \%)$ du massif de Ngovayang sont remarquablement proches de celles avancées par White (1983) pour l'ensemble de la région phytogéographique. Cet auteur affirme que la flore guinéo-congolaise dans son ensemble est remarquablement pure, avec plus de $80 \%$ d'endémiques et seulement $10 \%$ environ d'espèces de liaison. Cette prédominance d'espèces guinéo-congolaises dans les parcelles étudiées est largement imputable au fait que nous avons sélectionné des parcelles de forêts matures, peu ou pas perturbées par les activités humaines. Des résultats similaires ont été obtenus pour la flore du Kasaï, en République Démocratique du Congo (Lubini 2001) et pour la flore du centre du Gabon (Doucet 2003 ), avec respectivement $79 \%$ et $81 \%$ d'espèces guinéocongolaises.

Par ailleurs, la présence d'espèces appartenant aux familles des Huaceae, Hoplestigmataceae, Medusandraceae, Lepidobotryaceae, Octoknemaceae, et Scytopetalaceae, familles strictement liées au centre régional d'endémisme guinéo-congolais (White 1983), permet de rattacher sans ambigüité le massif de Ngovayang à ce fond floristique. Les forêts de basse et moyenne altitudes du massif comprennent $81,5 \%$ des familles ( 31 familles sur 38 ) et $39 \%$ de genres (19 genres sur 49) cités par White (1983) comme endémiques de ce centre régional d'endémisme.

La prédominance des espèces du domaine guinéen inférieur (tableau 1) dans la flore du massif de Ngovayang montre que ce dernier appartient bien au domaine guinéen

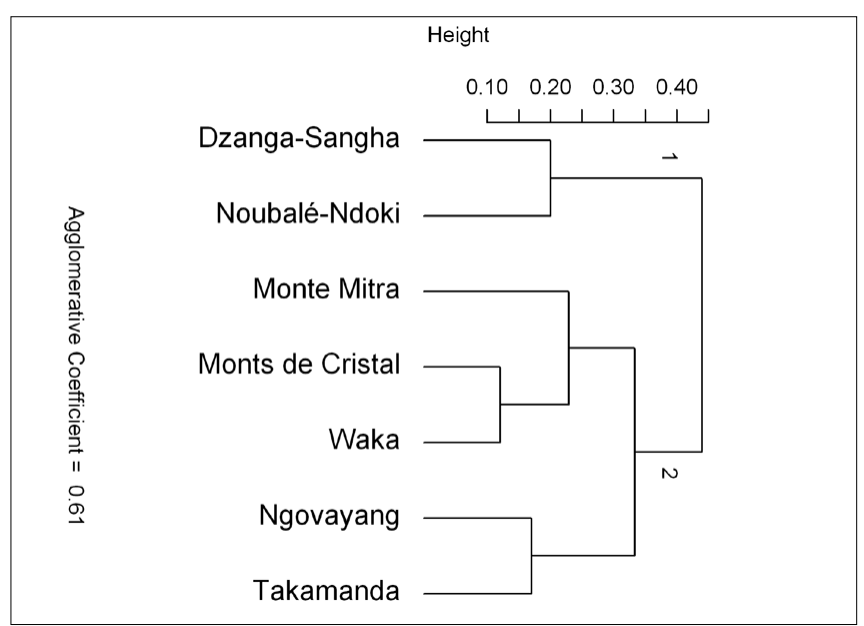

Figure 3 - Distances écologiques (Bray-Curtis binaire) entre les sites en Afrique Centrale sur la base des listes de familles. Le chiffre 1 représente les sites continentaux, et le chiffre 2 correspond aux sites côtiers. inférieur tel que défini par Aubréville (1962) et White (1979). Ce secteur phytogéographique est particulièrement soumis à la mousson atlantique ainsi que, dans sa partie sud, à l'effet refroidissant du courant du Benguela qui assure une humidité atmosphérique importante même en saison sèche (Suchel 1972, White 1983). Ce climat, favorable au développement des forêts denses sempervirentes, a pu perdurer par le passé et favoriser le développement et l'extension d'espèces caractéristiques sur l'ensemble de ce secteur basguinéen (Maley \& Brenac 1998). C'est le cas, par exemple, des Leguminosae-Caesalpinioideae. Plus de $40 \%$ des Leguminosae-Caesalpinioideae recensées à Ngovayang appartiennent au domaine guinéen inférieur (fig. 4). Parmi ces espèces, certaines ont été identifiées comme bio-indicatrices de forêts anciennes, voire de refuges forestiers, dans la région de Campo Ma'an, à environ $75 \mathrm{~km}$ au sud de Ngovayang (fig. 1; Tchouto et al. 2009). Il s'agit de Berlinia craibiana, B. auriculata, Gilbertiodendron ogoouense, Guibourtia tessmannii, Amphimas ferrugineus, Plagiosiphon longitubus et Leonardoxa africana subsp. gracilicaulis.

Les Leguminosae-Caesalpinioideae grégaires sont bien représentées dans tout le domaine guinéen inférieur, en particulier les espèces dont les mécanismes de dispersion (barochore et autochore) ne permettent pas une dispersion rapide à grande distance. C'est le cas, par exemple, des forêts de Korup (Newbery et al. 2004), de Campo-Ma'an (Tchouto et al. 2009), et de Ngovayang (Gonmadje et al. 2011). Seul le maintien de conditions favorables pendant des périodes longues peut expliquer leur répartition dans toute la région. Parmi ces espèces, on peut citer Tetraberlinia bifoliolata, Guibourtia tessmannii et Didelotia letouzeyi, qui dominent les forêts de Ngovayang et sont marqueurs des forêts sempervirentes côtières (Vivien \& Faure 1985, Wieringa 1999, Doucet 2003). Ainsi, la dominance des Leguminosae-Caesalpinioideae confirme l'appartenance du massif de Ngovayang au "district atlantique biafréen riche en Caesalpiniaceae" tel que défini par Letouzey $(1968,1985)$ sur des caractères qualitatifs (listes d'espèces) ou semi-quantitatifs (estimation à vue du grégarisme des espèces).

En revanche, les espèces à large distribution, qui illustrent le caractère secondaire ou de substitution d'un site donné consécutivement aux activités humaines (Evrard 1968, Habiyaremye 1997), sont faiblement représentées dans les forêts non perturbées de Ngovayang ( $2 \%$ des espèces). Cela confirme la maturité des peuplements étudiés. Nos relevés, bien que ne renfermant que très peu d'espèces de végétation secondaire, restent parmi les plus riches des forêts de la région (Gonmadje et al. 2011).

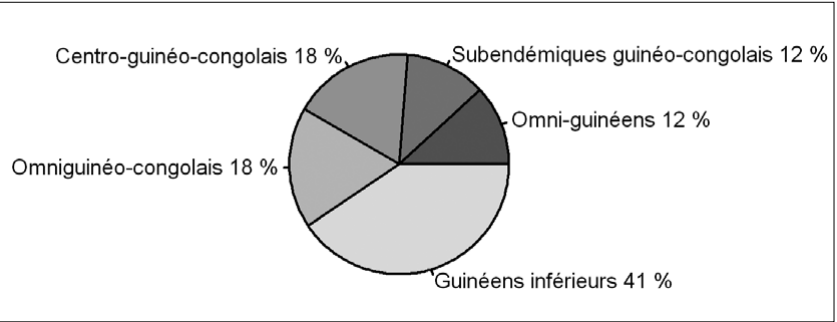

Figure 4 - Types phytogéographiques des LeguminosaeCaesalpinioideae inventoriées dans le massif de Ngovayang. 


\section{Dissimilarités floristiques entre les sites côtiers et conti- nentaux}

L'analyse phytogéographique des différents sites étudiés confirme l'originalité floristique des forêts situées sur le pourtour du golfe de Guinée comparativement à celles de l'intérieur du continent, y compris celles situées en marge de la Cuvette congolaise, comme Nouabalé-Ndoki et DzangaSangha. Ces résultats corroborent ceux d'Aubréville (1962), Letouzey $(1968,1985)$, White (1983), Senterre (2005) et Droissart (2009). Sur ces sites continentaux on constate, par exemple, une forte régression des Burseraceae et des Olacaceae (Senterre \& Nguema 2001, Sonké 2005, Sunderland \& Balinga 2005, Balinga et al. 2006).

Les différences climatiques présentes ou passées pourraient expliquer cette différenciation floristique ouest-est. Les sites côtiers, sous l'influence de la mousson atlantique, sont caractérisés par de fortes précipitations ( $\geq 2000 \mathrm{~mm} / \mathrm{an}$ ), une forte nébulosité et une saison sèche moins marquée (1-3 mois). En revanche, les sites continentaux sont déterminés par des précipitations plus faibles (1400-1700 mm/an) et une saison sèche plus longue (1-4 mois) et plus sévère, notamment en périphérie de la cuvette congolaise (Suchel 1972, White 1983). Les variations passées du climat ont aussi été probablement plus importantes pour Nouabalé-Ndoki et Dzanga-Sangha que pour les sites côtiers (Maley 2002). En effet, ces deux sites sont situés dans le corridor de la Sangha, une zone connue pour avoir subi une ouverture de la forêt et une extension de la savane durant les phases climatiques arides passées (White 1979).

D'autres facteurs, tels que le sol ou d'anciens impacts humains pourraient aussi expliquer ces différences floristiques (Boulvert 1986, Sheil 2001, Tuomisto et al. 2003, Réjou-Méchain et al. 2008). Ce dernier facteur a été minimisé autant que possible en écartant de nos analyses les parcelles inventoriées dans des forêts perturbées. Concernant le facteur sol, les parcelles sur sol inondable ou à nappe phréatique proche de la surface ont été écartées mais, il est possible que des différences dans la teneur en sable des parcelles de Nouabalé-Ndoki et Dzanga-Sangha (Boulvert 1983) puissent expliquer une partie de leur dissimilarité floristique relativement aux sites atlantiques, tous situés sur des sols nettement argileux. En l'absence de données précises sur les sols de ces sites, il nous est impossible d'en tirer une conclusion fiable. La multiplication de comparaisons de parcelles et des analyses plus fines intégrant la composition des sols seront nécessaires afin d'affiner notre compréhension des facteurs de variations biogéographiques sur toute la région.

\section{Affinités et dissimilarités floristiques entre les sites côtiers}

Les forêts côtières ou atlantiques ont en commun, parmi les taxa dominants, la sous-famille des Leguminosae-Caesalpinioideae et, les familles des Olacaceae, des Euphorbiaceae, des Clusiaceae, des Burseraceae, des Annonaceae et des Rubiaceae (Sunderland et al. 2003, 2004, Balinga et al. 2005, Balinga 2006, Gonmadje et al. 2011). Ceci expliquerait en partie la forte similarité floristique observée entre ces sites côtiers au niveau familial. L'importance des LeguminosaeCaesalpinioideae, en particulier les espèces grégaires, permet de rassembler ces forêts dans un même domaine phytogéographique: le domaine guinéen inférieur. Toutefois, des différences de composition floristique, tant au niveau spécifique que familial, ont permis d'identifier deux groupes de sites, Takamanda et Ngovayang d'une part, et les autres sites côtiers de l'autre (figs $2 \& 3$ ).

Contrairement à l'une des hypothèses émises au début de cet article, les forêts de Ngovayang ont ainsi plus d'affinités avec celles de Takamanda qu'avec les autres forêts bordant, au Sud, l'océan Atlantique. La présence de la LVC et du fleuve Sanaga semble jouer un rôle moins important dans la structuration phytogéographique des forêts de basse et moyenne altitude de Basse-Guinée que la 'charnière climatique' qui marque le passage du régime climatique boréal au régime austral (Suchel 1990, Thomas \& Goudie 2000); cette charnière climatique étant située au Sud du Cameroun, vers la frontière avec la Guinée-Equatoriale (fig. 1). De nombreux travaux ont montré que certains fleuves (Sanaga, Cross, Congo) jouent un rôle important de barrière géographique dans la structuration de la biodiversité animale; c'est le cas pour le Bonobo (Ericksson et al. 2004), le Gorille (Anthony et al. 2007) et d'autres primates (Colyn et al. 1991). Concernant la végétation, ce rôle de barrière de dispersion est beaucoup plus rarement évoqué que dans le règne animal mais pourrait toutefois fonctionner pour certaines espèces telles que Garcinia preussii (Clusiaceae) et Psorospermum membranaceum (Hypericaceae; Cheek et al. 2001), les genres Diospyros (White 1978) ou Begonia (Sosef 1994), et pour les Orchidaceae (Droissart 2009). Il ne semble toutefois pas s'appliquer aux peuplements d'arbres que nous avons étudiés.

Le massif de Ngovayang est géographiquement plus proche de Monte Mitra et des monts de Cristal que de Takamanda (fig. 1). Par ailleurs, la réserve de Takamanda est géographiquement plus proche de la LVC que du massif de Ngovayang. Certaines espèces de montagne, issues d'un fond floristique original et présentant des affinités avec l'Afrique orientale, auraient pu donner naissance à des formes de basse et moyenne altitude (White 1987), favorisant l'originalité du peuplement forestier de Takamanda. Mais ce mécanisme potentiel ne semble pas avoir fonctionné de cette manière, tout au moins concernant le peuplement des arbres. La réserve de Takamanda, située à la frontière nigéro-camerounaise, présenterait des affinités floristiques plus importantes avec les forêts du sud-est du Nigéria (White 1979, Letouzey 1985). Cependant, nos résultats montrent que, malgré la distance géographique qui les sépare, ainsi que leur position opposée vis-à-vis de la LVC et du fleuve Sanaga, Ngovayang et Takamanda sont floristiquement plus proches entre eux qu'ils ne le sont des forêts situées au sud de la charnière climatique (Monte Mitra, monts de Cristal, Waka). Le massif de Ngovayang constituerait alors une zone de transition floristique entre les forêts du Nigéria et celles s'étendant du sud-ouest Cameroun jusqu'en RDC.

La charnière climatique évoquée ci-dessus est le reflet du décalage des saisons entre les deux régimes climatiques boréal et austral. En réduisant le succès de la pollinisation (phénologie de floraison décalée) ou de l'établissement des plantules (dispersion et germination des graines hors la saison optimale), ce décalage pourrait compliquer, après une 
péjoration climatique, la recolonisation par certaines espèces forestières adaptées à l'un ou l'autre climat. L'hétérogénéité floristique observée au sein des sites côtiers serait due non seulement à la présence actuelle de cette charnière climatique mais aussi aux variations climatiques passées (Maley 1996, Plana et al. 2004). L'impact de ces fragmentations, en particulier celles du Pléistocène et de l'Holocène, a été conditionné par les réponses des différentes espèces à cette charnière climatique, qui peut ainsi favoriser la rémanence de ces anciennes fragmentations. Ceci pourrait expliquer la dissimilarité floristique existante entre les forêts de Ngovayang et de Takamanda avec celles situées plus au Sud, suggérant l'existence des deux entités phytogéographiques au sein du domaine de Basse-Guinée. Ces termes de charnière climatique (climatic hinge en anglais), bien que peu usités, matérialisent la ligne imaginaire qui coupe la zone tropicale en séparant les régions recevant l'essentiel des précipitations au cours de l'été boréal de celles qui sont affectées par les pluies d'été de l'hémisphère austral (Thomas \& Goudie 2000). Suchel (1990) a utilisé l'expression "ligne de démarcation du passage du régime boréal au régime austral" pour matérialiser cette zone de basculement climatique. Nous suivrons ainsi les appellations de ces deux auteurs, en évitant d'introduire dans le texte des termes qui - s'ils s'avèrent pratiques à utiliser - n'en sont pas moins sources de confusions. En effet, par assimilation à l'équateur géographique (cercle terrestre de latitude $0^{\circ}$ ), les termes $d$ ' "équateur climatique" ou "équateur thermique" ont été utilisés par plusieurs auteurs (Daïnou et al. 2010, Debout et al. 2011, Duminil et al. 2010), pour qualifier cette charnière climatique. L" "équateur thermique" est la zone des plus hautes températures moyennes autour de la terre, dont la position peut varier selon les époques mais qui a été situé, sur le long terme, à près de $5^{\circ}$ de latitude Nord, au nord de la zone de basculement climatique à laquelle nous faisons référence (Allaby \& Allaby 1999). L' "équateur climatique" correspond quant à lui à une ligne imaginaire où se rencontrent les masses d'air boréale et australe; cet équateur climatique varie au cours des saisons en fonction du balancement de la zone de convergence intertropicale (ZCIT). Si ces positions extrêmes sont mentionnées dans les travaux consultés (Beltrando \& Camberlin 1993, Allaby \& Allaby 1999, Kaser 1999), l'équateur climatique n'est jamais matérialisé par une ligne précise. Ce terme pourrait être pratique mais il s'avère finalement inadapté à rendre compte de la réalité géographique du basculement des saisons entre les deux hémisphères.

A l'échelle régionale, Balas et al. (2007) ont montré que la région du Sud-Cameroun, et celle formée par la GuinéeEquatoriale et le Gabon, sont deux régions climatiques bien distinctes, chacune étant homogène en termes de variabilité interannuelle des précipitations, de saisons humide et sèche principales. Au nord, la saison sèche principale est centrée sur décembre-février, avec des précipitations importantes en été boréal. Au sud de cette charnière climatique, la saison sèche principale se situe en juin-août; un creux de précipitation est plus ou moins marqué en hiver boréal mais les pluies restent toujours relativement importantes. Par ailleurs, Suchel (1990) a bien mis en évidence de manière détaillée le phénomène d'inversion des saisons sèches dans le sud-ouest du Cameroun. Le passage du régime boréal au régime austral, encore discret sur le territoire camerounais, devient plus visible un peu plus au sud de Ngovayang, en bordure de l'océan, notamment au sud de Campo, et s'étend en Guinée-Equatoriale et au Gabon. D'autres paramètres climatiques, plus discrets que la pluviométrie, ont aussi révélé un contraste Nord/Sud. Il s'agit des moyennes des températures minimales journalières, de l'humidité relative et de l'évaporation. D'après Suchel (1990), la ligne de démarcation du passage au véritable régime austral, située à environ $2^{\circ} 0$ ' $\mathrm{N}$, écorne l'angle sud-ouest du territoire camerounais (fig. 1).

D'autres paramètres importants pour la végétation différencient les climats observés au nord et au sud de cette charnière climatique. L'harmattan, un vent desséchant soufflant du nord-est en provenance du Sahara, atteint parfois la zone des forêts ombrophiles situées au nord de la charnière climatique mais ne pénètre pas dans les forêts du Sud. Au Ghana, par exemple, lorsque l'harmattan souffle, l'humidité relative peut tomber jusqu'à 53\% (White 1983). Le climat de l'hémisphère Nord est donc caractérisé par une saison sèche principale plus sévère, plus ensoleillée et plus sèche que celle du Sud (Suchel 1972, 1990, Saint-Vil 1984). La principale saison sèche australe est caractérisée quant à elle par une couverture nuageuse stratiforme - due à l'effet refroidissant du courant du Benguela - qui provoque une diminution des températures, limite l'évapo-transpiration et favorise les brouillards matinaux (Saint-Vil 1984, Suchel 1990, Fontaine \& Bigot 1993, Wotling et al. 1995, Bigot et al. 1997, Balas et al. 2007). Le climat y reste relativement humide et donc favorable à la survie des végétaux, même si les précipitations annuelles sont moins importantes qu'autour de la LVC.

Par ailleurs, plusieurs climatologues et palynologues (Bond et al. 1997, deMenocal et al. 2000, Leuschner \& Sirocko 2000, Maley 2002, Giresse et al. 2005) ont pu reconstituer les principales variations de la position de la charnière climatique durant l'Holocène, qui se situait plus au nord qu'actuellement, suggérant une remontée vers le nord de celle-ci. Au niveau du Cameroun, au cours de l'Holocène inférieur et moyen, la limite nord de la forêt dense se situait sur le rebord septentrional du plateau de l'Adamaoua. L'aire de distribution d'Aucoumea klaineana, dont la limite nord actuelle correspond à la charnière climatique, s'étendait durant l'Holocène inférieur et moyen jusqu'au bord du lac Ossa, $170 \mathrm{~km}$ au nord de sa limite actuelle (Reynaud-Farrera 1995). Le recul d'Aucoumea klaineana vers le Sud serait associé à la phase de péjoration climatique intervenue entre 2500 et $2000 \mathrm{BP}$. Le positionnement actuel de la charnière climatique daterait de l'Holocène récent (Bond et al. 1997, deMenocal et al. 2000, Leuschner \& Sirocko 2000, Maley 2002, Giresse et al. 2005). Le phénomène d'inversion des saisons et la présence d'une saison sèche plus drastique dans l'hémisphère Nord et l'histoire climatique passée dont il a été fait mention pourraient ainsi être la cause de la limitation à la dispersion de certaines espèces du Sud vers le Nord telle que Aucoumea klaineana (Muloko 2001, Born 2007), ou de différences génétiques au sein d'une même espèce: c'est le cas d'Irvingia gabonensis (Irvingiaceae; Lowe et al. 2010), Milicia excelsa (Moraceae; Daïnou et al. 2010) et Distemonanthus benthamianus (Léguminosae-Caesalpinioideae; Debout et al. 2011). 
Outre les Leguminosae-Caesalpinioideae, d'autres familles telles que les Olacaceae et les Burseraceae caractériseraient les forêts atlantiques matures (Doumenge 1992, Senterre \& Lejoly 2001, Doucet 2003), bien que leur importance soit variable en fonction des forêts considérées. C'est le cas des Burseraceae, qui sont moins importantes (en termes de FIV) dans les forêts camerounaises que dans celles de Guinée-Equatoriale, du Gabon ou du Congo (Doumenge 1992, Senterre \& Nguema 2001, Doucet 2003). En effet, les aires de répartition de plusieurs Burseraceae forestières sont, soit limitées au sud de l'équateur thermique, en climat d'hémisphère Sud, soit ne l'atteignent ou ne le dépassent que très marginalement, au Sud-Cameroun. C'est le cas d'Aucoumea klaineana et de diverses espèces de Dacryodes. Même parmi les Leguminosae-Caesalpinioideae, certaines espèces sont limitées au sud de la charnière climatique ( $A n$ thonotha ferruginea, A. mouandzae, A. pellegrinii, A. stipulacea, A. trunciflora) et d'autres au Nord (Anthonotha wijmacampensis et A. xanderi; Breteler 2010).

Bien que Droissart (2009) ait considéré la chaîne Ngovayang-Mayombe (Cameroun-RDC) comme une seule aire d'endémisme morcelée pour la distribution des Orchidaceae en Afrique Centrale, ses travaux ont aussi révélé, au sein des sites côtiers de Basse-Guinée qu'il a étudiés, l'existence de deux ensembles phytogéographiques qui corroborent nos résultats: un groupe formé par Ngovayang et Campo d'une part, et un autre formé par les monts de Cristal, le Monte Alen et le massif du Chaillu d'autre part. Même si l'auteur n'a pas expliqué l'existence de ces deux entités phytogéographiques, les différences constatées pourraient être liées à l'influence de la charnière climatique nord-sud.

$\mathrm{Au}$ total, et même si les mécanismes biologiques de différenciation floristique nord-sud ne sont pas encore totalement élucidés, l'importance de cette charnière climatique dans la structuration de la biodiversité végétale apparaît comme un élément de la biogéographie des forêts denses humides africaines qui a été par trop négligé jusqu'à présent. Nos propres données, combinées aux résultats présentés sur d'autres groupes biologiques (herbacées, arbustes) et à d'autres échelles taxonomiques (phylogéographie intraspécifique), fournissent ainsi des pistes pour la réinterprétation phytogéographique du domaine bas-guinéen.

\section{CONCLUSION}

Les forêts de basse et moyenne altitude du massif de Ngovayang présentent une composition floristique caractéristique de forêts atlantiques matures riches en LeguminosaeCaesalpinioideae grégaires, typiques du domaine floristique bas-guinéen. Ce massif constitue une zone de transition entre l'ensemble des forêts périphériques aux reliefs de la LVC et l'archipel de petits sommets qui bordent l'océan Atlantique jusqu'à la RDC. Cependant, le massif de Ngovayang semble floristiquement plus proche des forêts de basse et moyenne altitude Camerouno-nigériane que de celles de la Guinée Equatoriale et du Gabon. Il semble donc, comme cela a aussi été démontré par des travaux de phylogéographie, que la présence de la charnière climatique, aux confins camerouno-gabonais, ait constitué une barrière climatique suffisante pour permettre la différenciation d'assemblages floristiques particuliers au nord et au sud de cette limite.

Des études plus poussées sont toutefois nécessaires afin de préciser si ce schéma identifié sur les peuplements d'arbres des forêts de basse et moyenne altitude se confirme pour d'autres types biologiques (arbustes, lianes, épiphytes) et d'autres types forestiers, en particulier les forêts submontagnardes. Enfin, il sera nécessaire de compléter non seulement les inventaires sur les sites présentés ici, mais aussi de compléter l'échantillonnage par des inventaires similaires dans d'autres sites, permettant de mieux couvrir la variabilité des forêts du domaine guinéen inférieur. Ces données permettront d'apporter plus de précisions sur la phytogéographie et l'histoire de ces forêts, fournissant des informations nécessaires aux politiques et aux gestionnaires pour la mise en place des stratégies de conservation et de gestion des ressources forestières efficaces à l'échelle nationale, voire sousrégionale. Elles pourront également permettre le développement d'une meilleure compréhension de la paléo-histoire des forêts de cette partie du continent.

\section{MATERIEL SUPPLEMENTAIRE}

Le matériel supplémentaire est disponible au format pdf sur Plant Ecology and Evolution, Supplementary Data Site (http://www.ingentaconnect.com/content/botbel/plecevo/ supp-data), et comprend: (1) une liste des espèces d'arbres inventoriées et identifiées jusqu'au niveau spécifique dans le massif de Ngovayang; (2) une liste des espèces citées dans le texte, et absentes du massif de Ngovayang; et (3) les formules de calcul de l'Importance Value Index (IVI; Curtis \& McIntosh 1951), du Family Importance Value Index (FIV; Mori et al. 1983) et de l'indice de de dissimilarité de BrayCurtis binaire $\left(\mathrm{BC}_{\mathrm{ij}}\right)$.

\section{REMERCIEMENTS}

Nous remercions le projet "Sud Expert Plantes" (SEP) financé par le Ministère français des Affaires Etrangères et Européennes, et le Centre de Coopération Internationale en Recherche Agronomique pour le Développement (CIRAD) pour avoir financé ce travail. Nous remercions aussi N. Fauvet, du CIRAD, pour son assistance technique dans la cartographie. Nous ne manquerons pas de témoigner notre reconnaissance envers M. Sainge, P. Mezili and J.-M. Onana pour leur expertise dans l'identification des échantillons aussi bien sur le terrain qu'à l'Herbier National du Cameroun. Nous remercions également V. Freycon, D. McKey, J. Maley, B. Sinsin et les trois relecteurs anonymes pour leurs critiques et suggestions qui ont permis d'améliorer la qualité de ce travail. Nous ne saurons terminer sans remercier les populations locales du massif de Ngovayang qui ont contribué à la collecte des données sur le terrain.

\section{BIBLIOGRAPHIE}

Allaby A., Allaby M. (1999) "Thermal equator". A Dictionary of Earth Sciences. Encyclopedia.com. Available from http://www. encyclopedia.com/ [accessed 27 Jan. 2012]. 
Anthony N.M., Johnson-Bawe M., Jeffery K., Clifford S.L., Abernethy K.A., Tutin C.E.,Lahm S.A., White L.J.T., Utley J.F., Wickings E.J., Bruford M.W. (2007) The role of Pleistocene refugia and rivers in shaping gorilla genetic diversity in Central Africa. Proceedings of the National Academy of Sciences of the United States of America 104: 20432-20436. http://dx.do1. org/10.1073/pnas.0704816105

Aubréville A. (1958) A la recherche de la forêt en Côte d'Ivoire. Bois et Forêts des Tropiques 57: 12-27.

Aubréville A. (1962) Position chorologique du Gabon. In: Flore du Gabon 3: 3-11. Paris, Muséum National d'Histoire Naturelle.

Aubréville A., Leroy J.-F. (1961-1992) Flore du Gabon. Paris, Muséum National d'Histoire Naturelle.

Aubréville A., Leroy J.-F. (1963-1978) Flore du Cameroun, vols 1-20. Paris, Muséum National d'Histoire Naturelle.

Balas N., Nicholson S.E., Klotter D. (2007) The relationship of rainfall variability in West central Africa to sea-surface temperature fluctuations. International Journal of Climatology 27: 1335-1349. http://dx.doi.org/10.1002/joc.1456

Balinga M.P.B. (2006) A vegetation assessment of the Waka National Park, Gabon. CARPE Report. Available from http:// carpe.umd.edu/carpedocs/index.php?tab $=4$ [accessed 21 May 2012].

Balinga M.P.B., Issembe Y.A., Sunderland T.C.H., Nzabi T., Obiang D., Nyangadouma R. (2005) Quantitative vegetation assessment of the Monte Mitra forest using 1 hectare biodiversity plots (BDPs). In: Sunderland T.C.H. (ed.) A biodiversity assessment of the Monte Mitra forest, Monte Alen National Park, Equatorial Guinea. Available from http://carpe.umd.edu/carpedocs/index.php?tab=4 [accessed 21 May 2012].

Balinga M.P.B., Sainge M., Fombod E. (2006) A preliminary assessment of the vegetation of the Dzanga Sangha protect area complex, Central African Republic. A biodiversity assessment of the Dzanga Sangha protect area complex. CARPE Report. Available from http://carpe.umd.edu/carpedocs/index. php?tab $=4$ [accessed 21 May 2012].

Beltrando G., Camberlin P. (1993) Interannual variability of rainfall in the Eastern Horn of Africa and indicators of atmospheric circulation. International Journal of Climatology 13: 533-546. http://dx.doi.org/10.1002/joc.3370130505

Bigot S., Camberlin P., Moron V., Richard Y. (1997) Structure spatial de la variabilité des precipitations en Afrique: une transition climatique à la fin des années 1960. Comptes Rendus de l'Académie des Sciences, sér. 2, 324: 181-188.

Bond G., Showers W., Cheseby M., Lotti R., Hajdas I., Bonani G. (1997) A pervasive millenial-scale cycle in North Atlantic Holocene and glacial climates. Science 278: 1257-1266. http:// dx.doi.org/10.1126/science.278.5341.1257

Born C. (2007) Diversité génétique et dynamique des forêts d'Afrique centrale - une étude multi-échelle de la structure de la diversité génétique d'un arbre pionnier, Aucoumea klaineana. Thèse de Doctorat, Université de Montpellier 2, Montpellier, France.

Boulvert Y. (1983) Carte Pédologique de la république centrafricaine à 1: 1000 000. Paris, ORSTOM.

Boulvert Y. (1986) Carte Phytogéographique de la république centrafricaine (feuille OUEST - feuille EST) à 1: 1000 000. Paris, ORSTOM

Breteler F.J. (2010) Revision of the African genus Anthonotha (Leguminosae, Caesalpinioideae). Plant Ecology and Evolution 143: 70-99. http://dx.doi.org/10.5091/plecevo.2010.369

Brouat C., McKey D., Douzery E. (2004) Differentiation in a geographic mosaic of plants coevolving with ants: phylogeny of the
Leonardoxa africana complex (Leguminosae: Caesalpinioideae) using using amplified fragment length polymorphism markers. Molecular Ecology 13: 1157-1171. http://dx.doi.org/10.1111/ j.1365-294X.2004.02113.x

Caballé G. (1978) Essai sur la géographie forestière du Gabon. Adansonia, sér. 2,17: 425-440.

Cheek M., Mackinder B., Gosline G., Onana J.-M., Achoundong G. (2001) The phytogeography and flora of western Cameroon and the Cross River-Sanaga River interval. Systematics and Geography of Plants 71: 1097-1100. http://dx.doi. org/10.2307/3668742

Colyn M.M., Gautier-Hion A., Verheyen W. (1991) A re-appraisal of paleoenvironmental history in central Africa: evidence for a major fluvial refuge in the Zaire Basin. Journal of Biogeography 18: 403-407. http://dx.doi.org/10.2307/2845482

Conservatoire et Jardin botaniques de la Villle de Genève and South African National Biodiversity Institute, Pretoria (2009) African flowering plants database. Available from http://www.ville-ge. $\mathrm{ch} / \mathrm{musinfo} / \mathrm{bd} / \mathrm{cjb} /$ africa [accessed 11 Nov. 2011].

Curtis J.T., McIntosh R.P. (1951) An upland forest continuum in the prairie-forest border region of Wisconsin. Ecology 32: 476496. http://dx.doi.org/10.2307/1931725

Daïnou K., Bizoux J.P., Doucet J.L., Mahy G., Hardy O.J, Heurtz M. (2010) Forest refugia revisited: nSSRs and cpDNA sequences support historical isolation in a wide-spread African tree with high colonization capacity, Milicia excelsa (Moraceae). Molecular Ecology 19: 4462-4477. http://dx.doi.org/10.1111/j.1365294X.2010.04831.x

Dallmeier F., Kabel M., Rice R. (1992) Methods for long-term biodiversity inventory plots in protected tropical forest. In: Dallmeier F. (eds) Long term monitoring of biological diversity in tropical areas: methods for establishment and inventory of permanent plots: 11-46. Paris, MAB Digest 11, UNESCO.

Debout G.D.G., Doucet J.-L., Hardy O.J. (2011) Population history and gene dispersal inferred from spatial genetic structure of a Central African timber tree, Distemonanthus benthamianus (Caesalpinioideae). Heredity 106: 88-99. http://dx.doi. org/10.1038/hdy.2010.35

deMenocal P., Ortiz J., Guilderson T., Sarnthein M. (2000) Coherent high and low latitude climate variability during the Holocene warm period. Science 288: 2198-2202. http://dx.doi. org/10.1126/science.288.5474.2198

Doucet J.L. (2003) L'alliance délicate de la gestion forestière et de la biodiversité dans les forêts du centre du Gabon. Thèse de doctorat, Faculté universitaire des sciences agronomiques de Gembloux, Gembloux, Belgique.

Doumenge C. (1992) La Réserve de Conkouati: Congo. Le secteur sud-ouest. Gland, UICN.

Doumenge C., Garcia Yuste J.E., Gartlan S., Langrand O., Ndinga A. (2001) Conservation de la biodiversité forestière en Afrique centrale atlantique: le réseau d'aires protégées est-il adéquat? Bois et Forêts des Tropiques 268: 5-28.

Doumenge C., Ndinga A., Fomete N.T., Tchanou Z., Micha O.V., Ona N.N., Bourobou B.H., Ngoye A. (2003) Conservation de la biodiversité forestière en Afrique centrale atlantique. II. Identification d'un réseau de sites critiques. Bois et Forêts des Tropiques 276: 43-58.

Droissart V. (2009) Etude taxonomique et biogéographique des plantes endémiques d'Afrique Centrale atlantique: le cas des Orchidaceae. Thèse de doctorat, Université Libre de Bruxelles, Bruxelles, Belgique.

Duminil J., Heuertz M., Doucet J.-L., Bourland N., Cruaud C., Gavory F., Doumenge C., Navascue M., Hardy O.J. (2010) 
CpDNA-based species identification and phylogeography: application to African tropical tree species. Molecular Ecology 19: 5469-5483. http://dx.doi.org/10.1111/j.1365294X.2010.04917.x

Eriksson J., Hohmann G., Boesch C., Vigilant L. (2004) Rivers influence the population genetic structure of bonobos (Pan paniscus). Molecular Ecology 13: 3425-3435. http://dx.doi. org/10.1111/j.1365-294X.2004.02332.X

Evrard C. (1968) Recherches écologiques sur les peuplements forestiers des sols hydromorphes de la Cuvette centrale congolaise. O.N.R.D./INEAC, série scientifique 110. Brussels, INEAC.

Fontaine B., Bigot S. (1993) West African rainfall deficit and sea surface temperatures. International Journal of Climatology 13: 271-285. http://dx.doi.org/10.1002/joc.3370130304

Franqueville A. (1973) Atlas Regional Sud-Ouest 1. République du Cameroun. Yaoundé, ORSTOM.

Gemerden B.S. van (2004) Disturbance, diversity and distributions in Central African rain forest. PhD thesis, Wageningen University, Wageningen, The Netherlands.

Giresse P., Malet J., Kossoni A. (2005) Sedimentary environmental changes and millenial climatic variability in a tropical shallow lake (Lake Ossa, Cameroun) during the Holocene. Palaeogeography, Palaeoclimatology, Palaeoecology 218: 257-285. http:// dx.doi.org/10.1016/j.palaeo.2004.12.018

Gonmadje C.F., Doumenge C., McKey D., Tchouto M.G.P., Sunderland T.C.H., Balinga M.P.B., Sonké B. (2011) Tree diversity and conservation value of the Ngovayang massif, Cameroon. Biodiversity and Conservation 20: 2627-2648. http://dx.doi. org/10.1007/s10531-011-0095-z

Habiyaremye M.K.F.X. (1997) Etude phytocoenologique de la dorsale orientale du lac Kivu (Rwanda). Annales des Sciences Economiques 24: 1-276.

Hall J.B., Swaine M.D. (1976) Classification and ecology of closedcanopy forest in Ghana. Journal of Ecology 64: 913-951. http:// dx.doi.org/10.2307/2258816

Hall J.B., Swaine M.D. (1981) Distribution and ecology of vascular plants in tropical rainforest. Forest vegetation in Ghana. The Hague, Dr. W. Junk Publisher.

Hallé N. (1961-1987) Flore du Gabon. Vols. 2-17. Paris, Muséum National d'Histoire Naturelle.

Hawthorne W.D. (1995) Ecological profiles of Ghanaian Forest Trees. Oxford, Oxford Forestry Institute.

Kaser G. (1999) A review of the modern fluctuations of tropical glaciers. Global and Planetary Change 22: 93-103. http://dx.doi. org/10.1016/S0921-8181(99)00028-4

Keay R.W.J., Hepper F.N. (1954-1972) Flora of West Tropical Africa $2^{\text {nd }}$ Ed. 3 Vols. London, Crown Agents.

Lebrun J. (1960) Sur la richesse de la flore de divers territoires africains. Bulletin de l'Académie Royale, Sciences Outre mer, nov, sér. 6: 669-690.

Lebrun J. (1961) Les deux flores d'Afrique tropicale. Académie Royale de Belgique, Mémoires 8, 32(6).

Lebrun J.P., Stork A.L. (1991, 1992, 1995, 1997) Enumération des plantes à fleur d'Afrique tropicale, 4 Vols. Genève, Conservatoire et Jardin botaniques de la ville de Genève.

Léonard J. (1965) Contribution à la subdivision phytogéographique de la région guinéo-congolaise d'après la répartition géographique d'Euphorbiaceae d'Afrique tropicale. Webbia 19: 627-649.

Letouzey R. (1968) Etude phytogéographique du Cameroun. Paris, Paul Lechevallier.
Letouzey R. (1985) Notice de la carte phytogéographique du Cameroun au 1: 500 000. 5 Vols. Toulouse, Institut de la carte Internationale de la Végétation.

Leuschner D.C., Sirocko F. (2000) The low-latitude monsoon climate during Dansgaard-Oeschger cycles and Heinrich events. Quaternary Science Reviews 19: 243-254. http://dx.doi. org/10.1016/S0277-3791(99)00064-5

Lowe A.J., Harris D.J., Dormontt E., Dawson I.K. (2010) Testing putative African tropical forest refugia using chloroplast and nuclear DNA phylogeography. Tropical Plant Biology 3: 50-58. http://dx.doi.org/10.1007/s12042-010-9045-2

Lubini A.C. (1997) La végétation de la Réserve de biosphère de Luki au Mayombe (Zaïre). Opera Botanica Belgica 10.

Lubini A.C. (2001) Analyse phytogéographique de la flore forestière du secteur du Kasai au Congo-Kinshasa. Systematics and Geography of Plants 71: 859-872. http://dx.doi. org/10.2307/3668724

Maley J. (1996) The African rain forest - main characteristics of changes in vegetation and climate from the Upper Cretaceous to the Quaternary. In: Alexander I.J., Swaine M.D.,Watling R. (eds) Proceedings of the Royal Society of Edinburgh-Essays on the Ecology of the Guinea-Congo Rain Forest: 31-37. Edinburgh, Royal Society of Edinburgh.

Maley J. (2002) A catastrophic destruction of African forests about 2,500 years ago still exerts a major influence on present vegetation formations. IDS Bulletin 33: 13-30. http://dx.doi. org/10.1111/j.1759-5436.2002.tb00003.x

Maley J., Brenac P. (1998) Vegetation dynamics, palaecoenvironments and climatic change in the forest of western Cameroon during the last 28,000 years B.P. Review of Palaeobotany and Palynology 99: 157-187. http://dx.doi.org/10.1016/S00346667(97)00047-X

McKey D. (2000) Leonardoxa africana (Leguminosae: Caesalpinioideae): a complex of mostly allopatric subspecies. Adansonia 22: $71-109$.

Monod T. (1957) Notes botaniques sur les îles de São Tomé et Principe. Bulletin de l'IFAN, série A, 1: 19-79.

Mori S.A., Boom B.M., Carvalino A.M., Dos Santos T.S. (1983) The ecological importance of Myrtaceae in an eastern Brazilian wet forest. Biotropica 15: 68-70. http://dx.doi.org/10.2307/2388002

Muloko N. (2001) Phylogéographie de Aucoumea klaineana (Burseraceae): apport des marqueurs génétiques. Thèse de doctorat, Université de Montpellier 2, Montpellier, France.

Newbery D.M., van der Burgt X.M., Newbery D.M. (2004) Structure and inferred dynamics of a large grove of Microberlinia bisulcata trees in central African rain forest: the possible role of periods of multiple disturbance events. Journal of Tropical Ecology 20: 1-13. http://dx.doi.org/10.1017/S0266467403001111

Oksanen J., Blanchet G., Kindt R., Legendre P., O'Hara R.B., Simpson G.L., Solymos P., Stevens M.H.H., Wagner H. (2010) Vegan:community Ecology Package. Version 1.17-3. Available from http://CRAN.R-project.org/package=vegan [accessed 11 Nov. 2011].

Olivry J.C. (1986) Fleuves et rivières du Cameroun. Collection Monographies Hydrologiques d'ORSTOM 9. Paris, MESRESORSTOM.

Plana V., Gascoigne A., Forrest L.L., Harris D., Pennington R.T. (2004) Pleistocene and pre-Pleistocene Begonia speciation in Africa. Molecular Phylogenetics and Evolution 31: 449-461. http://dx.doi.org/10.1016/j.ympev.2003.08.023

Rejou-Mechain M., Pelissier R., Gourlet-Fleury S., Couteron P., Nasi R., Thompson J.D. (2008) Regional variation in tropical forest tree species composition in the Central African Republic: 
an assessment based on inventories by forest companies. Journal of Tropical Ecology 24: 663-674. http://dx.doi.org/10.1017/ S0266467408005506

Reynaud-Farrera I. (1995) Histoire des paléoenvironnements forestiers du Sud-Cameroun à partir d'analyses palynologiques et statistiques de dépôts Holocènes et actuels. Thèse de doctorat, Université de Montpellier 2, Montpellier, France.

Saint-Vil J. (1984) La grande saison sèche au Gabon. Annales de 1'Université Nationale du Gabon 5: 107-119.

Senterre B. (2001) La phytogéographie des forêts denses de Guinée Equatoriale, avec une attention particulière aux forêts de Nsork. Systematics and Geography of Plants 71: 1087-1096. http:// dx.doi.org/10.2307/3668741

Senterre B. (2005) Recherches méthodologiques pour la typologie de la végétation et la phytogéographie des forêts denses d'Afrique tropicale. Université Libre de Bruxelles, Bruxelles, Belgique.

Senterre B., Lejoly J. (2001) Trees diversity in the Nsork rain forest (Rio Muni, Equatorial Guinea). Acta Botanica Gallica 148: 227-235.

Senterre B., Nguema N. (2001) La diversité des ligneux dans la forêt de Nsork (Guinée Equatoriale). Systematics and Geography of Plants 71: 837-846. http://dx.doi.org/10.2307/3668722

Sheil D. (2001) Long-term observations of rain forest succession, tree diversity and responses to disturbance. Plant Ecology 155: 183-199. http://dx.doi.org/10.1023/A:1013243411819

Soladoye M.O. (1985) A revision of Baphia (LeguminosaePapilionoideae). Kew Bulletin 40: 291-386. http://dx.doi. org/10.2307/4108263

Sonké B. (2005) Forêts de la Réserve du Dja (Cameroun): Etudes floristiques et structurales. Scripta Botanica Belgica 32: 144.

Sonké B., Dessein D., Taedoumg H., Groeninckx I., Robbrecht E. (2008) A new species of Colletoecema (Rubiaceae) from southern Cameroon with a discussion of relationships among basal Rubioideae. Blumea 53: 533-547. http://dx.doi. org/10.3767/000651908X607495

Sonké B., Nguembou K.C., Cheek M., Davis A.P. (2006a) A new species of Chassalia (Rubiaceae, Rubioideae) from southern Cameroon: C. bipindensis. Kew Bulletin 61: 585-589.

Sonké B., Nguembou K.C., Davis A.P. (2006b) A new dwarf Coffea (Rubiaceae) from Southern Cameroon. Botanical Journal of the Linnean Society 151: 425-430. http://dx.doi.org/10.1111/ j.1095-8339.2006.00508.x

Sosef M.S.M. (1994) Refuge begonias: taxonomy, phylogeny and historical biogeography of Begonia sect. Loasibegonia and sect. Scutobegonia in relation to glacial rainforest refuges. Wageningen Agricultural University Papers 94: 1-306.

Suchel J.-B. (1972) La répartition des pluies et les régimes pluviométriques au Cameroun. Travaux et Documents de Géographie Tropicale. Bordeaux, Yaoundé, CEGET-CNRS.

Suchel J.-B. (1990) Les modalités du passage du régime climatique boréal au régime climatique austral dans le Sud-Ouest camerounais. Cahier du Centre de Recherche de Climatologie. Université de Bourgogne, Dijon 13: 63-76.

Sunderland T.C.H., Balinga M. (2005) Evaluation préliminaire de la végétation du Parc National de Noubalé-Ndoki et de sa zone tampon, Congo. CARPE Report. Available from http://carpe. umd.edu/carpedocs/index.php?tab=4 [accessed 21 May 2012].

Sunderland T.C.H., Comiskey J.A., Besong S., Mboh H., Fonwebon J., Dione M.A. (2003) Vegetation assessment of Takamanda Forest Reserve, Cameroon. In: Comiskey J.A., Sunderland T.C.H., Sunderland-Groves J.L. (eds) Takamanda: the Biodiver- sity of an African Rainforest. SIMAB Series 8: 19-53. Washington, Smithonian Institution.

Sunderland T.C.H., Walters G., Issembe Y. (2004) A preliminary vegetation assessment of the Mbé national park, monts de Cristal, Gabon. CARPE Report. Available from http://carpe.umd. edu/carpedocs/index.php?tab=4 [accessed 21 May 2012].

Tchouto M.G.P. (2004) Plant diversity in central African rain forest. Implications for biodiversity conservation in Cameroon. $\mathrm{PhD}$ thesis, Wageningen University, Wageningen, The Netherlands.

Tchouto M.G.P., De Wilde J.J.F.E., De Boer W.F., Van der Maesen L.J.G., Cleef A.M. (2009) Bio-indicator species and Central African rain forest refuges in the Campo-Ma'an area, Cameroon. Systematics and Biodiversity 7: 21-31. http://dx.doi. org/10.1017/S1477200008002892

Thomas D., Goudie A. (2000) The Dictionary of Physical Geography. $3^{\text {rd }}$ Ed. Oxford, Blackwell Publishing.

Tuomisto H., Ruokolainen K., Yli-Halla M. (2003) Dispersal, environment, and floristic variation of western Amazonian forests. Science 299: 241-244. http://dx.doi.org/10.1126/science. $107 \overline{8037}$

Versteegh C.P.C., Sosef M.S.M. (2007) Revision of the African genus Annickia (Annonaceae). Systematics and Geography of Plants 77: 91-118.

Vivien J., Faure J.J. (1985) Arbres des forêts denses d'Afrique Centrale. Paris, Ministère des Relations Extérieures, Coopération et Développement.

Waterloo M.J., Ntonga J.C., Dolman A.J., Ayangma A.B. (2000) Impact of shifting cultivation and selective logging on the hydrology and erosion of rain forest land in south Cameroon. Tropenbos-Cameroon Documents 3. Wageningen, TropenbosCameroon Programme, the Netherlands.

White F. (1978) The taxonomy, ecology and chorology of African Ebenaceae I. the Guineo-Congolian species. Bulletin du Jardin Botanique National de Belgique 48: 245-358.

White F. (1979) The Guinéo-Congolian region and its relationships to other phytochoria. Bulletin du Jardin Botanique National de Belgique 49: 11-55. http://dx.doi.org/10.2307/3667933

White F. (1983) The Vegetation of Africa. Paris, UNESCO.

White F. (1987) Long-distance dispersal and the origins of the Afromontane Flora. Sonderband der naturwissenschaftlichen Verein Hamburg 7: 87-116.

White L., Edwards A. (2001) Conservation en forêt pluviale africaine: Méthode de recherches. New York, Wildlife Conservation Society.

Wieringa J.J. (1999) Monopetalanthus exit. A systematic study of Aphanocalyx, Bikinia, Icuria, Michelsonia and Tetraberlinia (Leguminosae, Caesalpinioideae). $\mathrm{PhD}$ thesis, Wageningen University, Wageningen, The Netherlands.

Wilson E.O. (1995) The Diversity of Life. Cambridge, Harvard University Press.

Wotling G., Mahé G., Lebarbe L., L'Hote Y. (1995) Analyse par les vecteurs régionaux de la variabilité spatio-temporelle des précipitations annuelles liées à la mousson africaine. Veille Climatique Satellitaire, Lannion 52: 58-73.

Paper based on results presented during the XIX ${ }^{\text {th }}$ AETFAT Congress (Madagascar 2010). Manuscript received 29 Oct. 2010; accepted in revised version $23 \mathrm{Feb}$. 2012. This paper will be reprinted in the Proceedings of the XIX ${ }^{\text {th }}$ AETFAT Congress.

Communicating Guest Editor: Porter P. Lowry II. 